\title{
Modelica Delft-Tyre Interface
}

\author{
Edo Drenth \\ Magnus Gäfvert \\ Modelon AB \\ Ideon Science Park \\ Lund, Sweden \\ edo.drenth@modelon.se $\quad \underline{\text { magnus.gafvert@modelon.se }}$
}

\begin{abstract}
The TNO ${ }^{\mathrm{i}}$ Delft-Tyre is a renowned model for the pneumatic tire in the automotive industry based upon the famous Magic Formula first introduced by Bakker et.al. in the late eighties [1]. The name Magic Formula seems to appear first at the $1^{\text {st }}$ Delft colloquium on tires four years later [2]. The Magic Formulae themselves have evolved greatly during the last two decades with contributions from a wide variety of companies and researchers.

The Magic Formula is widely used in the automotive (and gaming) industry because of its ease of use to represent the complicated tire characteristics.

TNO has marketed the Magic Formula tire model as Delft-Tyre and implemented the dynamic forces and moments computation routines, including the extension of SWIFT [3], in a variety of multibody simulation packages, like ADAMS and DADS and general purpose simulation software Simulink ${ }^{\mathrm{ii}}$.

Modelon has in close cooperation with TNO by means of extensive benchmarks implemented the MF-Tyre/MF-Swift in Modelica. This paper presents the work conducted to implement the TNO tire models in Modelica which now is available as a commercial library in Dymola ${ }^{\text {iii }}$.
\end{abstract}

Keywords: Delft-Tyre, semi-empirical tire model, Magic Formula, SWIFT-Tyre

\section{Introduction}

Modelica is gaining popularity as a modeling and simulation language. In order to further increase the number of possible applications by means of lowering the threshold to embark on the Modelica route, an interface to TNO's MF Delft-Tyre/MF Swift has been developed. Rather than following the objectoriented modeling path stipulated in [5], this interface creates a single wheel class that includes tire characteristics and road-tire interface. One of the advantages using this solution, over the referenced one, is the well proven underlying code for force and moment computation of the tire characteristics. The tire model is semi-empirical and widely used in the automotive industry due to its ease of use and correlation with measured characteristics. Also, the necessary tire data is relatively easy and commercially available.

\section{The models}

\subsection{Wheel model}

The tire model used is TNO's MF-Tyre/MF-Swift. The MF-Tyre is solely based on the Magic Formula and can be used in models that are used for low frequency analysis $(<8 \mathrm{~Hz})$, like exploring handling characteristics of a vehicle. The MF-Swift, that requires a separate license from TNO, can be used in models that are intended to be used in a higher frequency range up to $100 \mathrm{~Hz}$, and thus useful in for example ride and controller development studies. The MFSwift tire model includes a rigid ring that models the tire belt and introduces additional rigid body modes to represent tire dynamics.

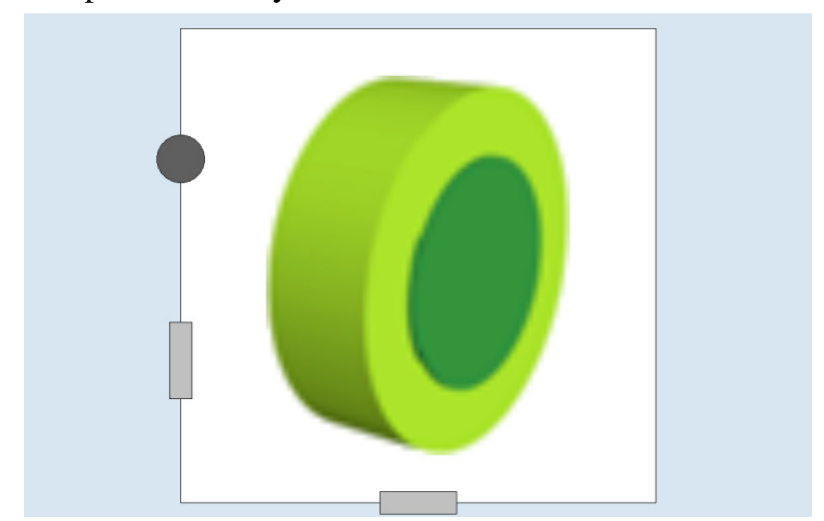

Figure 1 Wheel Model in Dymola (preferred) 
The Modelica interface is firstly made available in Dymola and other simulation environments are in progress. In Dymola a wheel model (see Figure 1) is made available that incorporates the MF-Tyre/MFSwift.

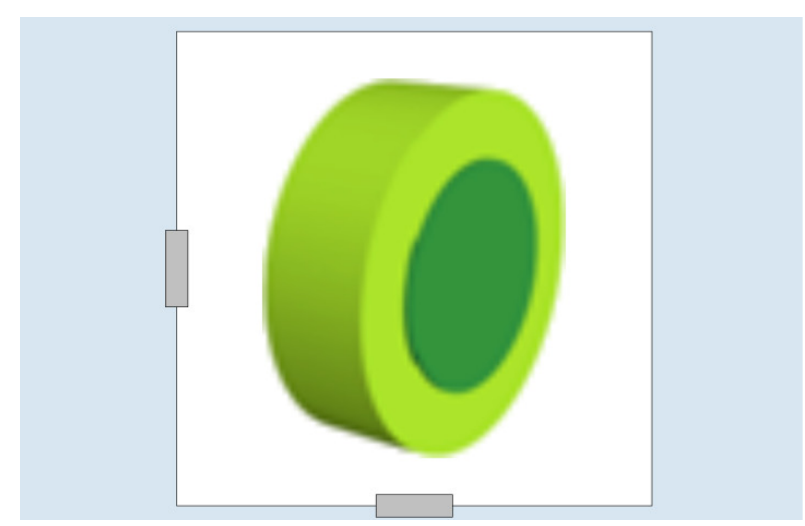

Figure 2 Wheel Model in Dymola

The preferred wheel model utilizes Dymola's capabilities of combining 1-DOF and 3-DOF multibody mechanics. The wheel is mounted with two connectors; a flange for the wheel spin degree of freedom and a normal connector for the wheel hub orientation. This is a numerical efficient method to include a power train in the complete vehicle models and compatible with the wheel implementation in Dymola Vehicle Dynamics Library, although not required for the Dymola Delft-Tyre interface.

TNO's Application Programming Interface, API, does allow for different types of interface and the straight forward interface with only one connector is also made available (see Figure 2). In this particular case the reference frame spins around the wheel spin axis.

\subsection{Moving road}

The API of MF-Tyre/MF-Swift allows, among different standard supplied types of road, moving roads. Moving roads are used to simulate for example a four-poster rig where the tire 'road' interface is moving vertically. Therefore a second connector is introduced in the wheel model that connects the wheel to the moving road and only visible when moving road is selected in the parameter dialogue.

\section{Benchmark}

\subsection{Introduction}

TNO has developed MF-Tyre/MF-Swift interfaces to three different simulation programs themselves. The results of these three interfaces have been thoroughly tested with help of simple models and smart load cases to exercise all functionality and features. The entire virtual test program is specified in [7].

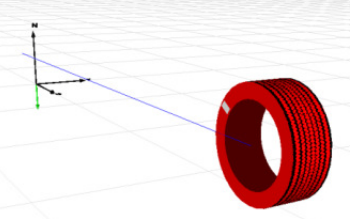

Figure 3 Single Wheel Model

In order to be allowed to release the MF-Tyre/MFSwift interface for Modelica, sanctioned by TNO Automotive, models had to be made and run in Dymola according to the referenced specification.

The single wheel model (see Figure 3) is run many different ways in order to explore and verify the MFTyre/MF-Swift robustness and accuracy. For example, the tire model can be run in a steady-state mode, such that the relaxation length is omitted. In case of a motor cycle tire (with motor cycle tire data) the wheel model is run with significant camber velocity as well as spin velocity resulting in large camber to road angles.

Also, a simple vehicle model (see Figure 4) is simulated to extend the virtual tire tests. Modeling the ply-steer and especially conicity, the tires will have to be identified to be left- or right hand mounted.

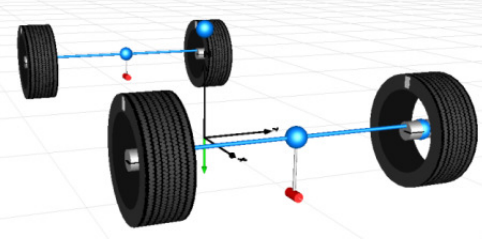

Figure 4 Simple Vehicle Model

The vehicle model will also be used on a four poster rig to verify the moving road interface. This four poster rig at the same time also verifies that the implemented tire model gives adequate results at standstill.

\subsection{Execution}

The created models discussed in the chapter above had to be executed through the entire virtual extensive test program specified in [7]. The simulation results for all the different test runs will have to be 
forwarded to TNO in a readable format. TNO will compare all results with their base line results with help of an automated process. The feedback by means of many graphical plots is sent back to the issuer of the simulation results. Never is the base line disclosed in numerical readable format. Once the simulation results match (some exceptions allowed, see chapter below) with the base line results, the interface is deemed to be implemented correctly. Experiences have shown this is a tedious process.

\subsection{Results}

During the course of executing the benchmarks and discussing and looping the results with TNO it became apparent that the specification in some instances was not concise enough and different interpretations could be made. Through discussion consensus was reached in all cases. In some cases the Dymola models had to be adjusted in other cases the specification had to be updated in order to aid future benchmarks with other simulation programs.

For instance the benchmark specification expected so called 'cut-forces' and 'cut-torques' between two bodies, where this has neither standard support in Modelica nor in Dymola. Specialized multibody simulation programs may have functionality to sum all forces and torques between two bodies, which becomes equivalent to cut-forces and cut-torques between two bodies. Cut-force and cut-torque sensors had to be created and inserted in the models at the right places.

Another discrepancy was found in one of the tests where the simple vehicle model had to perform a brake action on a split friction road surface. The benchmark specified a brake torque profile as function of time, but it became apparent that a special filtering function was used in the base line models to accommodate brake torques (see chapter below for detailed discussion).

\section{Brake model}

\subsection{Introduction}

As indicated in the chapter above, a discrepancy was found between the base line vehicle model and the Dymola vehicle model. The brake torque capacity was specified as function of time and an example of the front wheel brake torque capacity is depicted in Figure 5. However, the actual brake torque is basically limited by the actual surface friction between tire and road and the actual vertical tire force. Hence, the actual resulting brake torque may be lower than the brake torque capacity, which is determined by the clamp forces, disc and pad friction and effective disc radius (in case of a disc brake).

\subsection{Actual brake torque}

Mathematically the brake torque is a result of the brake torque capacity and the external load on the wheel by means of longitudinal tire forces. The longitudinal forces on the tire are dependent on the load case as vertical tire force, actual surface friction and kinetic energy of the vehicle that the tire supports.

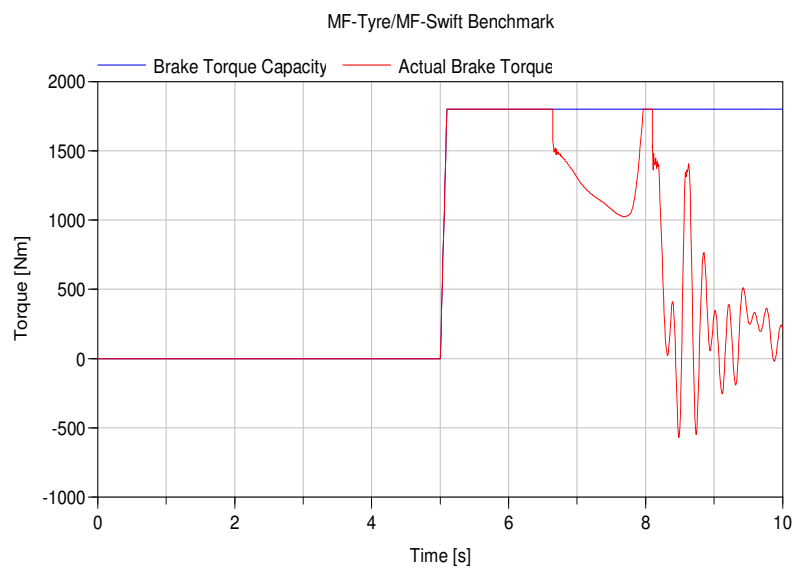

Figure 5 Brake torque capacity (blue) and actual brake torque (red) on front left wheel

The direction of the brake torque is also dependent on the load case and in its simplest form the brake torque takes the form:

$$
T_{b r}=T_{\text {cap }} \operatorname{sign}\left(\omega_{\text {wheel }}\right)
$$

In many simulation programs the sign of the wheel spin velocity will cause numerical difficulties. Hence smoother sign functions are used. For example:

$$
T_{b r}=T_{\text {cap }} \tanh \left(\omega_{\text {wheel }}\right)
$$

Of course such solution will distort the actual result. Modelica allows, as a standard feature, for accurate friction torque modeling as discussed in [8].

\subsection{Results}

Figure 5 through Figure 7 show some Dymola results of the actual benchmark with the split friction braking maneuver. 


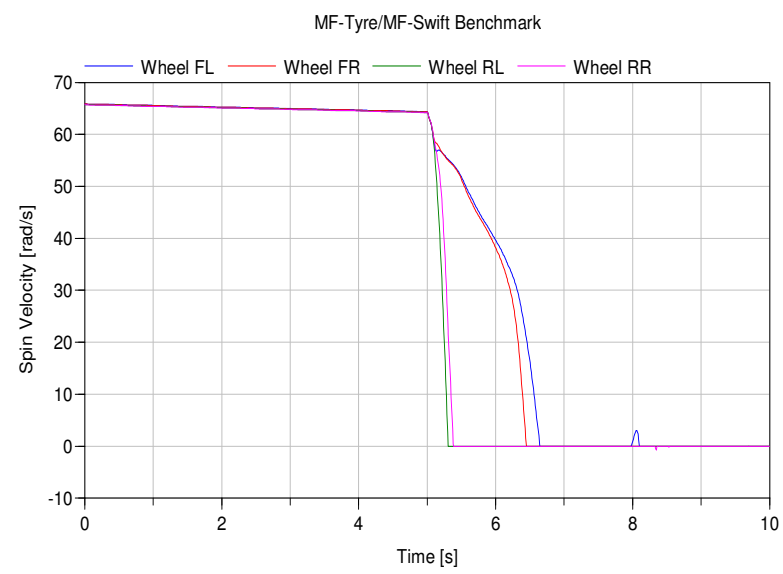

Figure 6 Wheel spin velocity traces

Figure 5 shows a trace of the brake torque capacity and the actual brake torque of the front left wheel. It can clearly be seen that the brake locks after about 6.5 seconds of simulation time, because the actual brake torque is lower than the brake torque capacity. At around 8 seconds of simulation time the brake unlocks a short while because the brake torque capacity and actual brake torque are equal.

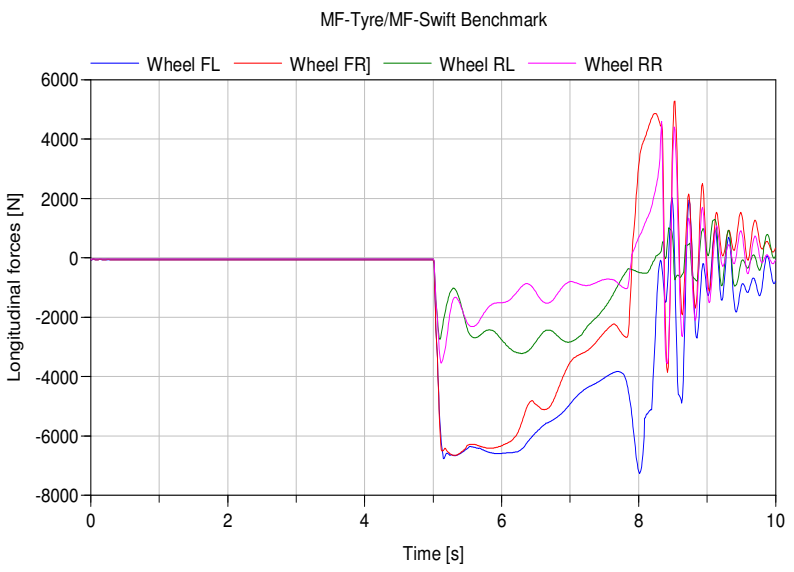

Figure 7 Traces of longitudinal tire forces

These results are also supported in the wheel spin velocity traces of Figure 6 . The wheel spin velocity of the front left wheel becomes zero indeed after approximately 6.5 seconds of simulation time. Also, at around 8 seconds of simulation time the wheel spins up a split second.

For sake of reference the tire longitudinal force traces are shown in Figure 7. The vehicle will actually spin due to the yaw moment disturbance as a result of the split surface friction and the brake forces may switch sign.

The shown results deviate from the base line simulations run in non-Modelica based other simulation software as soon as one of the braked wheels locked, but were deemed correct due to modeling differences.

\section{Conclusions}

The extensive benchmark conducted in order to approve the MF-Tyre/MF-Swift Modelica interface has proven Modelica based simulation software Dymola to be a great tool to perform vehicle dynamics analyses. Yet with access to the renowned Magic Formula based tire model as a commercial library. This interface will leverage Modelica as simulation language for vehicle dynamics studies.

\section{Outlook}

Other Modelica simulation software solutions are in progress to adopt this interface.

\section{References}

[1] E.Bakker, L. Nyborg, H.B.Pacejka, Tyre Modelling for Use in Vehicle Dynamics Studies; SAE 870421, 1987.

[2] H.B. Pacejka, E.Bakker, The Magic Formula Tyre Model, $1^{\text {st }}$ International Colloquium on Tyre Models for Vehicle Dynamcis Analysis, Delft, The Netherlands, 1991

[3] J.J.M. van Oosten, H.B. Pacejka, SWIFTTyre: An accurate tyre model for ride and handling studies also at higher frequencies and short road wavelengths, proceedings International ADAMS User Conference, Orlando, USA, 2000

[4] H.B.Pacejka, Tire and Vehcile Dynamics, Butterworth-Heinemann, Oxford, 2002

[5] M. Andres, D. Zimmer, F.E. Cellier, ObjectOriented Decomposition of Tire Characteristics based on semi-empirical Models, proceedings of $7^{\text {th }}$ International Modelica Conference, Como, Italy, 2009

[6] MF-Tyre/MF-Swift 6.1.2 Help Manual, TNO Automotive, The Netherlands, 2010

[7] MF-Tyre/MF-Swift Tyre Benchmarks, revision 1.3, TNO Automotive, The Netherlands, 2010

[8] M. Otter, H.Elmqvist, Modeling and Realtime Simulation of an Automatic Gearbox using Modelica, $9^{\text {th }}$ European Simulation Symposium, Passau, Germany, 1997

i TNO - Netherlands Organisation for Applied Scientific Research 
ii ADAMS, DADS and Simulink are trademarks of their respective owners

iii' Dymola is a Dassault Systèmes Modelica based simulation software 